\title{
Classification for Avian Malaria Parasite Plasmodium Gallinaceum Blood Stages by Using Deep Convolutional Neural Networks
}

\section{Veerayuth Kittichai}

King Mongkut's Institute of Technology Ladkrabang

Morakot Kaewthamasorn

Chulalongkorn University

\section{Suchansa Thanee}

Chulalongkorn University

Rangsan Jomtarak

Suan Dusit University

\section{Kamonpob Klanboot}

King Mongkut's Institute of Technology Ladkrabang

\section{Kaung Myat Naing}

King Mongkut's Institute of Technology Ladkrabang

\section{Teerawat Tongloy}

King Mongkut's Institute of Technology Ladkrabang

\section{Santhad Chuwongin}

King Mongkut's Institute of Technology Ladkrabang

Siridech Boonsang ( $\nabla$ siridech.bo@kmitl.ac.th )

King Mongkut's Institute of Technology Ladkrabang https://orcid.org/0000-0003-2866-8902

\section{Research}

Keywords: Plasmodium gallinaceum, Malaria, Rapid screening, Classification, Deep learning

Posted Date: March 11th, 2021

DOl: https://doi.org/10.21203/rs.3.rs-282527/v1

License: (c) (1) This work is licensed under a Creative Commons Attribution 4.0 International License. Read Full License

Version of Record: A version of this preprint was published at Scientific Reports on August 19th, 2021. See the published version at https://doi.org/10.1038/s41598-021-96475-5. 

10 *Correspondence:

11 Siridech.bo@kmitl.ac.th

$12{ }^{5}$ Department of Electrical Engineering, Faculty of Engineering, King Mongkut Institute of 13 Technology Ladkrabang, Bangkok, Thailand 


\section{Abstract}

28 Background: The infections of an avian malaria parasite (Plasmodium gallinaceum) in domestic chickens presents a major threat to poultry industry because it cause economical loss in both quality and quantity of meat and egg productions. Deep learning algorithms have been developed to identify avian malaria infections and classify its blood stage development.

Methods: In this study, four types of deep convolutional neural networks namely Darknet, Darknet19, darknet19_448x448 and Densenet 201 are used to classify $P$. gallinaceum blood stages. We randomly collected dataset of 10,548 single-cell images consisting of four parasite stages from ten-infected blood films stained by Giemsa. All images were confirmed by three welltrained examiners.

37 Results: In the model-wise comparison, the four neural network models gave us high values in the mean average precision at least 95\%. Darknet can reproduce a superior performance in classification of the P. gallinaceum development stages across any other model architectures. In addition, Darknet also has best performance in multiple class-wise classification, scoring the average values of greater than 99\% in accuracy, specificity, sensitivity, precision, and F1-score.

42 Conclusions: Therefore, Darknet model is more suitable in the classification of P. gallinaceum

43 blood stages than the other three models. The result may contribute us to develop the rapid 44 screening tool for further assist non-expert in filed study where is lack of specific instrument for 45 avian malaria diagnostic.

Keywords: Plasmodium gallinaceum, Malaria, Rapid screening, Classification, Deep learning 


\section{Background}

Avian malaria, a mosquito-borne disease, is one of the most common veterinary threats in tropical regions, including South East Asia and South Asia [1]. Plasmodium gallinaceum is an important causative agent of avian malaria, which causes more than $80 \%$ mortalityif left untreated [2]. The disease entails an economic and agricultural loss in poultry processes systems such as poor quality and quantity of meat and egg production [1]. Early and rapid routine screening for a safe and low-cost parasite infection may help prevent transmissions of the disease. Microscopic inspection is a gold standard approach and is widely used to examine and identify avian malariainfected blood stages under thin-blood film examinations, the outcome of which is validated by highly trained and qualified technicians [3]. Nevertheless, the precise outcome of the abovementioned procedure depends on the consistency of blood smearing and staining process. In addition, asymptomatic with low parasite infection can be time-consuming and may be undetectable or questionable as a result of inter/intra examiner variability [3]. In addition, unspecific clinical symptoms of avian malaria, such as anorexia, anemia, and green stools, are often seen [1,4]. While most of the nucleic acid-based amplification process, such as the polymerase chain reaction (PCR) assay, is an efficient method with a high sensitivity and malaria detection specificity, it requires an optimal consistency of genomic DNA and expensive tools such as thermocycler and electrophoresis apparatus [5-7]. A professional molecular biologist is also required for further verification of the analysis. Consistently, the molecular biology approach could not always be affordable in low-income and resource-limited nations.

Artificial intelligence (AI) technology is currently a positive integration success in a diverse area of interest, including agriculture, medicine and veterinary medicine [8-15]. However, the methods are combined and tailored for individual datasets that differ in size of the region of interest. In specific, deep learning technology is applied to end-to-end methods of extraction of features and self-discovery. Computer-aided machine learning algorithms, AI subdivision, have been developed to identify human malaria infections and to classify their blood stage of the parasite growth. These can be used to assist clinical decision-making. Machine learning applications have also been studied in the documented veterinary field [16]. The previous work demonstrated microplatforms to study and identify the infected avian red blood cell by using morphological modifications on the RBC surface to reveal the phases of $P$. gallinaceum [17]. Since malaria has been described as a disease of blood and blood-forming tissues, the blood film sample has been 
87 diagnosed to better understand different degrees of disease [18]. Early rapid screening of parasite

88 infection with reliable and also low cost development is required, which could help us deter the

89 spread of the disease. Therefore, timely identification of malaria parasite in a blood smear test is

90 crucial because it needs reliable and early diagnosis for successful containment [19].

91 Deep learning is a revolutionary and groundbreaking approach that has been developed to 92 incorporate a microscopic analysis for the veterinary medicine field. Previous research have 93 suggested a diagnostic tool in veterinary medicine focused on image analysis using machine 94 learning techniques [20-22], such as fish disease diagnosis based microscopic method [23]. The 95 analysis refered to above is intended to enhance the inspection of the pathogen region in the image 96 by means of object detection, which includes various techniques, including noise reduction, edge 97 detection, morphological operations and context extraction. In addition, several neural network 98 models have been used to contend with the animal sector, such as the support vector machine 99 (SVM) [24, 25], Single-Shot MultiBox Detector (SSD) model used to evaluate the percentage of 100 reticulocytes in cat's samples [26], Alexnet for classification of fish disease [27]. The works 101 described above makes it possible to apply deep learning algorithms in the field of veterinary 102 medicine.

103 The deep learning algorithm is very popular and useful with the emergence of a high-power 104 computing machine to be used to study the classification of images and the recognition of clinical 105 issues. Previously, several techniques for image-based identification and classification of malaria 106 parasite infections have been discussed, such as dark stretching technique [28, 29], modified fuzzy 107 divergence technique [30], segmentation techniques [19], adaptive color segmentation and tree108 based decision classification [31], segmentation, feature extraction, and SVM classifier [32], 109 convolutional neural classification [33, 34]. Moreover, deep CNN research have been conducted 110 under the channel color space segmentation method [35], deep belief network technique [36], the 111 Faster Region-based Convolutional Neural Network (Faster R-CNN) [37], and the MATLAB112 based Zach Threshold process for segmentation technique [38]. Successfully, several studies have 113 reported that the use of deep learning models to classify malaria parasites as automatic, rapid, and 114 accurate approaches [39-41]. Interestingly, more than 95\% of the accuracy is recorded in the 115 detection of malaria-infected cells using three well-known CNNs, including LeNet, AlexNet, and 116 GoogLeNet [42]. 
Vijayalakshmi and Kanna (2020) used VGG19-SVM to detect high-performance infected 118 and non-infected malaria parasite images as follows: 93.44 per cent sensitivity; 92.92 per cent 119 specificity; 89.95 per cent precision, 91.66 per cent F-score and 93.13 per cent accuracy [43]. 120 Many CNNs have been continuously created in the last year following the application of LeNet, 121 AlexNet, GoogLeNet and VGG. CNN has also been developed to provide a number of pre-trained 122 ImageNet classification models [44], including AlexNet, Darknet, VGG-16, Resnet [45], ResNext, 123 and Densenet201 [46], for use as effective in image classification applications. According to an 124 evaluation of the performance of these pre-trained models, Densenet201 has a higher accuracy 125 than Darknet. Nevertheless, Darknet provides the fastest processing time in both CPU and GPU. 126 In the application area, Darknet19 obtained the best result in differentiating chest X-Rays of 127 COVID-19 patients from other bacterial and viral pneumonias than all others of 16-deep CNNs 128 [47]. Although deep learning approaches have been achieved to identify CT scans of the chest and 129 X-ray of the chest for diagnosis of pneumonia caused by coronavirus infection, Densenet201 is 130 more capable of classifying and detecting medical images than some well-known deep transfer 131 learning models; namely VGG16, Inception ResNet, Resnet 152v2, AlexNet, ResNet18 and 132 SqueezeNet [48].

133 In this work, we implement four types of deep convolutional neural networks, namely 134 Darknet, Darknet19, darknet19 448x448 and Densenet 201, to characterize the avian malaria blood stages of $P$. gallinaceum. We compare the output of the deep convolutional neural network model to facilitate the prediction of parasitized and normal chicken's RBCs in thin blood film photos.

137 The stage of malaria development is also diagnosed and infers the extent of infection as well as the risk of malaria transmissions. In addition, to author's knowledge, this research work is the first 140 datasets relevant to clinical problems and would be useful for the disease diagnosis method in the 141 poultry industry. 


\section{Materials and Methods}

\section{Data collections}

Archived Giemsa-stained thin-blood films were have been collected from previous studies $[1,49]$. The Protocol on Animal Use was approved by the Institutional Animal Care and Use Committee of the Faculty of Veterinary Science, Chulalongkorn University (No. 1931091). The parasite infection was confirmed by three well-trained investigators using a microscopic diagnosis. Ten $P$. gallinaceum-infected chicken blood films were randomly chosen. A total of 432 photographs of malarial developmental stages were captured at $1000 \mathrm{x}$ by oil immersion magnification using a light microscope (Olympus CX31, Tokyo, Japan). The photos were taken with Olympus DP21-SAL digital camera (Tokyo, Japan). Of which, an individual RBC picture was chosen to a photograph a blood film from a region of interest to the so-called monolayer area. Each single-parasitized RBC image has been individually cropped under the CIRA CORE framework module (Fig. 1). The dataset consists of 10,548 cell images with a significant proportion of regions of interest (ROI). Every class obtained from any picture was randomly divided into training ( 80 per cent) and testing ( 20 per cent) sets, minimizing potential similarities between these two sets. The physiological and morphological class labelling of the training collection was accompanied by 'normal RBC', 'trophozoite', 'schizont', and 'gametocyte', respectively. While disproportionate sample size between classes has been identified and can trigger biased-prognosis against a class with a large number of image sets, deep learning approach with multi-layered layers and data annotation can be explored prior to model training. To speed up model convergence, rescale the image from raw pixels to $448 \times 448$ pixels before training with

Figure 1. Prediction of the outcome of selectable models. The color of the guide map indicated the field of interest predicted by the model. The $\mathrm{X}$-axis is the raw picture as input and models such as Darknet, Darknet19, Darknet19_448 and Densenet201. Y-axes are individual chicken-blood

174 cells that are regular and those that are infected malaria, including trophozoites, schizonts and 175 gametocytes. 


\section{Model selection and data augmentation}

179 The selectable models used are the top ranking of the ILSVRC2016 ImageNet competition to 180 identify pathological tasks. These versions are in the following sizes; Densenet-201, Darknet, 181 Darknet-19 and Darknet-19 448x448. These models were preprocessed in the "DeepClassif" 182 module under the CiRA Core base (Fig. 2). In order to avoid over-fitting of the training picture 183 collection, the data augmentation was produced prior to training. Data augmentation techniques 184 involve rotations, brightness/ contrast, turning, blueness and Gaussian noise. In addition, the 185 trained models were early paused to register the learned parameters before the end of their training 186 loss of 100,000 epochs. The likelihood of a threshold greater than and equal to $50 \%$ is considered 187 to be a true positive value. The ROI was automatically masked and colored with a JET color map.

Figure 2. The schematic of the deep learning classification of the Darknet19 neural network model. Filters each convolution layer help generate feature map in the part of feature extraction. Guide map of the JET color gradient is used to visualize the input based on optimal weight.

These models mentioned above were trained on the LINUX operating system based Ubuntu version 16.04, 16 GB RAM, and NVIDIA GeForce GTX1070Ti graphic processor unit with less than $24 \mathrm{~h}$ of commuting time. The model configuration is performed in $\mathrm{C}++\mathrm{using}$ Keras's API with Tensorflow backend and CUDA/CuDNN dependencies.

\section{Model evaluations}

The performance quality of the model- and class-wise prediction were evaluated in terms of the following statistical metrics: accuracy, precision, sensitivity, specificity, misclassification rate and F1 score [50].

$$
\begin{aligned}
& \text { Precision }=\frac{T P}{T P+F P} \ldots . \\
& \text { Sensitivity }=\frac{T P}{T P+F N} . \\
& \text { Specificity }=\frac{T N}{T N+F P} .
\end{aligned}
$$

203

204

$$
\text { Accuracy }=\frac{T P+T N}{T P+T N+F P+F N}
$$




$$
\begin{aligned}
& \text { Missclassification rate }=\frac{F P+F N}{T P+T N+F P+F N} \ldots \ldots \ldots \text { (5) } \\
& F 1 \text { score }=\frac{2 \times(\text { sensitivity } \times \text { precision })}{\text { sensitivity }+ \text { precision }} \ldots \ldots \ldots \ldots \ldots \text { (6) }
\end{aligned}
$$

where TP is the number of true positive values, $\mathrm{TN}$ is the number of true negative values, $\mathrm{FP}$ is the number of false positive values, and FN is the number of false negative values.

Confusion matrix table was created to demonstrate the overall accuracy of both the model and class classification. An area under the Receiver Operating Characteristic (ROC) curve (AUC) 213 at $95 \%$ confident of interval (CI) was designed to calculate the average accuracy of the model 214 using python software.

\section{Results}

\section{Comparison of neural network model performance}

218 In this analysis, model-wise performance was assessed as to whether the model was the 219 best-selected model based on a guide map and used to estimate P. gallinaceum-infected blood 220 phases. These models included Darknet, Darknet-19, Darknet-19_448 and Densenet201 as 221 described above. These simulations were calculated and compared the robustness of the neural 222 network models for the classification of avian malaria parasite stages using average accuracy, uncertainty confusion matrix table and ROC curve. In addition, we randomly split the single-cell images for the training and testing of the image sets to determine whether the different models chosen would produce the same classification outcome. Each single-cell image may be viewed in either a training/ validation or a test range. All well networks have offered us high average accuracy values of over 95 per cent, which is up to 99 per cent for the darknet algorithm (Table 1). General accuracy from the confusion matrix table of the darknet model showed a better value than 97 per cent as comparison to every other model (Table 2). Specifically, this manifested in an overall AUC ranking of 0.986 - 1.000 (Fig. 3). Overall, the darknet models can reproduce superior performance in the classification of the development phases of $P$. gallinaceum in every other model architecture. 
235 Table 1. Model-wise comparison based on precision per class and the average.

\begin{tabular}{l|ccccc}
\hline \multicolumn{1}{c|}{ DCNNs } & Mean average precision & Normal & Trophozoite & Schizont & Gametocyte \\
\hline Darknet & 0.990 & 0.993 & 0.968 & 1.000 & 1.000 \\
Darknet19 & 0.990 & 0.992 & 0.966 & 1.000 & 1.000 \\
Darknet19_448 & 0.983 & 0.966 & 0.965 & 1.000 & 1.000 \\
Densenet201 & 0.954 & 0.964 & 0.956 & 0.896 & 1.000 \\
\hline
\end{tabular}

237 Table 2. Comparison of the studied model performance using the confusion matrix table based 238 on the test image set.

\begin{tabular}{l|cccc}
\hline \multicolumn{1}{c|}{ Darknet } & Normal & Trophozoite & Schizont & Gametocyte \\
\hline Normal & $97.38 \%$ & $0.83 \%$ & $0.00 \%$ & $0.00 \%$ \\
Trophozoite & $2.62 \%$ & $99.17 \%$ & $0.00 \%$ & $0.00 \%$ \\
Schizont & $0.00 \%$ & $0.00 \%$ & $100.00 \%$ & $0.00 \%$ \\
Gametocyte & $0.00 \%$ & $0.00 \%$ & $0.00 \%$ & $100.00 \%$ \\
\hline
\end{tabular}

\begin{tabular}{l|cccc}
\hline \multicolumn{1}{c|}{ Darknet19 } & Normal & Trophozoite & Schizont & Gametocyte \\
\hline Normal & $97.21 \%$ & $1.03 \%$ & $0.00 \%$ & $0.00 \%$ \\
Trophozoite & $2.79 \%$ & $98.97 \%$ & $0.00 \%$ & $0.00 \%$ \\
Schizont & $0.00 \%$ & $0.00 \%$ & $100.00 \%$ & $0.00 \%$ \\
Gametocyte & $0.00 \%$ & $0.00 \%$ & $0.00 \%$ & $100.00 \%$ \\
\hline
\end{tabular}

\begin{tabular}{l|cccc}
\hline \multicolumn{1}{c|}{ Darknet19_448 } & Normal & Trophozoite & Schizont & Gametocyte \\
\hline Normal & $97.21 \%$ & $4.34 \%$ & $0.00 \%$ & $0.00 \%$ \\
Trophozoite & $2.79 \%$ & $95.66 \%$ & $0.00 \%$ & $0.00 \%$ \\
Schizont & $0.00 \%$ & $0.00 \%$ & $100.00 \%$ & $0.00 \%$ \\
Gametocyte & $0.00 \%$ & $0.00 \%$ & $0.00 \%$ & $100.00 \%$ \\
\hline
\end{tabular}

\begin{tabular}{l|cccc}
\hline \multicolumn{1}{c|}{ Densenet201 } & Normal & Trophozoite & Schizont & Gametocyte \\
\hline Normal & $96.56 \%$ & $4.55 \%$ & $0.00 \%$ & $0.00 \%$ \\
Trophozoite & $3.44 \%$ & $94.01 \%$ & $0.00 \%$ & $0.00 \%$ \\
Schizont & $0.00 \%$ & $1.45 \%$ & $100.00 \%$ & $0.00 \%$ \\
Gametocyte & $0.00 \%$ & $0.00 \%$ & $0.00 \%$ & $100.00 \%$ \\
\hline
\end{tabular}


239 Figure 3. Comparison of the model- and class-wise performance based on AUC under the ROC

240 curve

241

\section{Multiple class comparison}

243 According to the process, we fed a single cell into the studied model and allowed it predicted the

244 result with the testing image set which is managed by experts. An observation of the classification

245 result gave an empirical result in discriminating the malaria parasite stages based on the best-

246 selected network model.

247 Although we trained the model with an unequal number of sample sizes between classes,

248 the multiple class-wise quality of the neural network model showed great statistical values for all 249 classes with greater than $99 \%$ of all performance matrices used without bias towards any class

250 (Table 3). In addition, our selected model can classify the malaria stages with a low rate of 251 misclassification less than $1 \%$. This may be the advantage of using the class-balancing (extra 252 function) before starting the model training within the CiRA CORE program. This study result 253 suggests the possible validation of the model with various blood smears obtained from real 254 situations.

Table 3. Different statistical metrics of darknet neural network models.

\begin{tabular}{l|cccccc}
\hline Class Name & Accuracy & $\begin{array}{c}\text { Misclassification } \\
\text { Rate }\end{array}$ & Sensitivity & Specificity & Precision & F1-Score \\
\hline Normal & 0.983 & 0.017 & 0.974 & 0.993 & 0.993 & 0.983 \\
Trophozoite & 0.983 & 0.017 & 0.992 & 0.976 & 0.968 & 0.980 \\
Schizont & 1.000 & 0.000 & 1.000 & 1.000 & 1.000 & 1.000 \\
Gametocytes & 1.000 & 0.000 & 1.000 & 1.000 & 1.000 & 1.000 \\
\hline \multicolumn{1}{c}{ Average } & $\mathbf{0 . 9 9 2}$ & $\mathbf{0 . 0 0 9}$ & $\mathbf{0 . 9 9 2}$ & $\mathbf{0 . 9 9 2}$ & $\mathbf{0 . 9 9 0}$ & $\mathbf{0 . 9 9 1}$ \\
\hline
\end{tabular}

Discussion

In this research, the robustness of deep neural network models leads to the development of new method for more rapid screening under a microscope of avian malaria. Specifically, asymptomatic infections that can lead to the spread of the disease and even cause mortality without well-planned prevention $[51,52]$. Though we have trained network models with a real patientlevel yet unbalanced size of each class, the performance of well-trained proposed models has 
264 shown an outstanding outcome based on many statistical parameters, including in a multi-class 265 comparison. Since the experiment proved to be very successful, it could contribute to the achievement of the development of innovative technology in order to apply and test it in a real

267 situation. Nevertheless, in the near future, the inter-and intra-variability examinations should be analyzed in order to approve the degree of agreement between the practical success measurements of the predictive model and the human expert before the realization of the technology [53].

Limitation of the study is deeply based on the determination of three key points for the preparation of the dataset [54], including 1) differences in the parasite-blood stages [55], 2) infection status that can induce either single or multiple infections, and 3) co-infections in any single blood cell. Another result of dataset planning, though less than those mentioned above is the imbalance data. In the case of differences in the blood stage of each parasite, an increasing number of sample is a potential solution, however the analysis has had an effect on model efficiency under the research design. This may be because the picture set is a special attribute between classes that helps to increase class differentiation for the well-trained models. In addition, we selected an image of almost a single stage of parasite infection to be analyzed. Since the staining of artefacts/impurities of uninfected blood cells can interfere with the prediction, the expert's simple staining of color under the Giemsa staining process also provides us with the ideal dataset [56]. Nevertheless, the model must be validated in a specific environment of multiple pathogens and co-infections, which could already exist in field samples.

\section{Conclusions}

Here, the application of a deep neural learning network to identify and verify avian malaria and also to assess it using truly invisible images that have never been published. Based on the findings of the report, our excellent CiRA CORE framework concept was demonstrated. Based on our results, we can infer that there is only a model learning approach and data preparation that should be used in the research design. Fortunately, the analysis revealed a high importance of different statistical measures as mentioned above, which could be of interest to decision-making help for cytological technicians. It would be useful to diagnosis malaria infections wherever it is 


\section{Abbreviations}

PCR: polymerase chain reaction; DNA: deoxyribonucleic acid; AI: artificial intelligence;

297 RBC: red blood cell; SVM: support vector machine; SSD: single-short multibox detector; Faster 298 R-CNN: faster region-based convolutional neural network; ROI: region of interest; DCNN: deep 299 convolutional neural network; TP: true positive; TN: true negative; FP: false positive; FN: false 300 negative; CI: confident interval; ROC: receiver operating characteristic curve; AUC: area under 301 the ROC curve.

302

\section{Acknowledgements}

We are grateful to Suchansa Thanee at the Parasitology Unit, Faculty of Veterinary 305 Science, Chulalongkorn University, for assisting in the parasite identification. We also thank the 306 College of Advanced Manufacturing Innovation, King Mongkut's Institute of Technology 307 Ladkrabang which provided the deep learning platform and software to support the research 308 project.

Author's contributions

SB, VK and SC conceived and designed the research study; SB, VK, MK, KMN and SC wrote the manuscript; VK, KMN, RJ, KK and SC performed the computational experiment; MK

314 SB read and approved the final manuscript.

\section{Data availability}

The data that support the findings of this study are available from the corresponding 318 author's GitHub repository: URL: https://git.cira-lab.com/cira-medical/classification-of-avian319 malaria-parasite

The Protocol on Animal Use was approved by the Institutional Animal Care and Use Committee of the Faculty of Veterinary Science, Chulalongkorn University (No. 1931091). 


\section{Consent for publication}

Not applicable

\section{Competing interest}

The authors declare that they have no competing interest.

\section{Author details}

${ }^{1}$ Faculty of Medicine, King Mongkut Institute of Technology Ladkrabang, Bangkok, Thailand.

${ }^{2}$ Veterinary Parasitology Research Unit, Faculty of Veterinary Science, Chulalongkorn

University, Bangkok, Thailand. ${ }^{3}$ Faculty of Science and Technology, Suan Dusit University,

Technology Ladkrabang, Bangkok, Thailand. ${ }^{5}$ Department of Electrical Engineering, Faculty of

Engineering, King Mongkut Institute of Technology Ladkrabang, Bangkok, Thailand

\section{Reference}

341 1. Pattaradilokrat S, Tiyamanee W, Simpalipan P, Kaewthamasorn M, Saiwichai T, Li J, et

342 al. Molecular detection of the avian malaria parasite Plasmodium gallinaceum in Thailand. Vet 343 Parasitol. 2015;210(1-2):1-9.

3442 . Permin A, Juhl J. The development of Plasmodium gallinaceum infections in chickens 345 following single infections with three different dose levels. Vet Parasitol. 2002;105(1):1-10.

346 3. Wongsrichanalai C, Barcus MJ, Muth S, Sutamihardja A, Wernsdorfer WH. A review of 347 malaria diagnostic tools: microscopy and rapid diagnostic test (RDT). Am J Trop Med Hyg. $348 \quad 2007 ; 77(6$ Suppl):119-27.

349 4. Williams RB. Avian malaria: clinical and chemical pathology of Plasmodium gallinaceum in the domesticated fowl Gallus gallus. Avian Pathol. 2005;34(1):29-47. 5. Miguel RB, Coura JR, Samudio F, Suarez-Mutis MC. Evaluation of three different DNA extraction methods from blood samples collected in dried filter paper in Plasmodium subpatent infections from the Amazon region in Brazil. Rev Inst Med Trop Sao Paulo. 2013;55(3). 6. Polley SD, Weedall GD, Thomas AW, Golightly LM, Conway DJ. Orthologous gene sequences of merozoite surface protein 1 (MSP1) from Plasmodium reichenowi and $\mathrm{P}$. gallinaceum confirm an ancient divergence of P. falciparum alleles. Mol Biochem Parasitol. 2005;142(1):25-31.

358 7. Polley SD, Mori Y, Watson J, Perkins MD, Gonzalez IJ, Notomi T, et al. Mitochondrial DNA targets increase sensitivity of malaria detection using loop-mediated isothermal amplification. J Clin Microbiol. 2010;48(8):2866-71. 
8. Carin L, Pencina MJ. On Deep Learning for Medical Image Analysis. JAMA. 2018;320(11):1192-3.

363 9. Kim K, Hyun J, Kim H, Lim H, Myung H. A Deep Learning-Based Automatic Mosquito Sensing and Control System for Urban Mosquito Habitats. Sensors (Basel). 2019;19(12). 10. Liu G, Nouaze JC, Touko Mbouembe PL, Kim JH. YOLO-Tomato: A Robust Algorithm for Tomato Detection Based on YOLOv3. Sensors (Basel). 2020;20(7).

11. Rajaraman S, Antani SK, Poostchi M, Silamut K, Hossain MA, Maude RJ, et al. Pretrained convolutional neural networks as feature extractors toward improved malaria parasite detection in thin blood smear images. PeerJ. 2018;6:e4568.

12. Shen D, Wu G, Suk HI. Deep Learning in Medical Image Analysis. Annu Rev Biomed Eng. 2017;19:221-48.

13. Unver HM, Ayan E. Skin Lesion Segmentation in Dermoscopic Images with Combination of YOLO and GrabCut Algorithm. Diagnostics (Basel). 2019;9(3).

14. Wang Q, Bi S, Sun M, Wang Y, Wang D, Yang S. Deep learning approach to peripheral leukocyte recognition. PLoS One. 2019;14(6):e0218808.

15. Zhuang Z, Liu G, Ding W, Raj ANJ, Qiu S, Guo J, et al. Cardiac VFM visualization and analysis based on YOLO deep learning model and modified 2D continuity equation. Comput Med Imaging Graph. 2020;82:101732.

16. Cihan P, Gökçe E, Kalipsiz O, editors. A Review of Machine Learning Applications in Veterinary Field2017.

17. Hsu Y, Lu P, Tang WC, editors. Microplatforms for avian malaria studies. 2011 6th IEEE International Conference on Nano/Micro Engineered and Molecular Systems; 2011 20-23 Feb. 2011.

18. Valkiūnas G, Iezhova TA. Keys to the avian malaria parasites. Malaria Journal. 2018;17(1):212.

19. Sheeba F, Thamburaj R, Mammen JJ, Nagar AK, editors. Detection of Plasmodium Falciparum in Peripheral Blood Smear Images2013; India: Springer India.

20. Brannon NG, Seiffertt JE, Draelos TJ, Wunsch DC, 2nd. Coordinated machine learning and decision support for situation awareness. Neural Netw. 2009;22(3):316-25.

21. Bertram CA, Gurtner C, Dettwiler M, Kershaw O, Dietert K, Pieper L, et al. Validation of Digital Microscopy Compared With Light Microscopy for the Diagnosis of Canine Cutaneous Tumors. Vet Pathol. 2018;55(4):490-500.

22. Awaysheh A, Wilcke J, Elvinger F, Rees L, Fan W, Zimmerman KL. Review of Medical Decision Support and Machine-Learning Methods. Vet Pathol. 2019;56(4):512-25.

23. Park J, Oh M, Han S, editors. Fish Disease Diagnosis System Based on Image Processing of Pathogens' Microscopic Images. 2007 Frontiers in the Convergence of Bioscience and Information Technologies; 2007 11-13 Oct. 2007.

24. Wan L, Bao W, editors. Animal Disease Diagnoses Expert System Based on SVM2010; Berlin, Heidelberg: Springer Berlin Heidelberg.

25. Malik S, Kumar T, Sahoo AK, editors. Image processing techniques for identification of fish disease. 2017 IEEE 2nd International Conference on Signal and Image Processing (ICSIP); 2017 4-6 Aug. 2017.

26. Vinicki K, Ferrari P, Belic M, Turk R. Using Convolutional Neural Networks for Determining Reticulocyte Percentage in Cats2018 March 01, 2018:[arXiv:1803.04873 p.]. Available from: https://ui.adsabs.harvard.edu/abs/2018arXiv180304873V. 
27. Waleed A, Medhat H, Esmail M, Osama K, Samy R, Ghanim TM, editors. Automatic Recognition of Fish Diseases in Fish Farms. 2019 14th International Conference on Computer Engineering and Systems (ICCES); 2019 17-17 Dec. 2019. 28. Kaewkamnerd S, Uthaipibull C, Intarapanich A, Pannarut M, Chaotheing S, Tongsima S. An automatic device for detection and classification of malaria parasite species in thick blood film. BMC Bioinformatics. 2012;13 Suppl 17(Suppl 17):S18-S.

29. Hanif NSMM, Mashor MY, Mohamed Z, editors. Image enhancement and segmentation using dark stretching technique for Plasmodium Falciparum for thick blood smear. 2011 IEEE 7th International Colloquium on Signal Processing and its Applications; 2011 4-6 March 2011. 30. Ghosh M, Das D, Chakraborty C, Ray AK, editors. Plasmodium vivax segmentation using modified fuzzy divergence. 2011 International Conference on Image Information Processing; 2011 3-5 Nov. 2011.

31. Pamungkas A, Adi K, Gernowo R. Identification of plasmodium falciparum development phase in malaria infected red blood cells using adaptive color segmentation and decision tree based classification. International Journal of Applied Engineering Research. 2015;10:4043-56. 32. Preedanan W, Phothisonothai M, Senavongse W, Tantisatirapong S, editors. Automated detection of plasmodium falciparum from Giemsa-stained thin blood films. 2016 8th International Conference on Knowledge and Smart Technology (KST); 2016 3-6 Feb. 2016. 33. Gopakumar G, Swetha M, Siva GS, Subrahmanyam GRKS. Automatic detection of Malaria infected RBCs from a focus stack of bright field microscope slide images. Proceedings of the Tenth Indian Conference on Computer Vision, Graphics and Image Processing; Guwahati, Assam, India: Association for Computing Machinery; 2016. p. Article 16.

34. Liang Z, Powell A, Ersoy I, Poostchi M, Silamut K, Palaniappan K, et al., editors. CNNbased image analysis for malaria diagnosis. 2016 IEEE International Conference on Bioinformatics and Biomedicine (BIBM); 2016 15-18 Dec. 2016.

35. Haryanto SEV, Mashor MY, Nasir ASA, Jaafar H, editors. A fast and accurate detection of Schizont plasmodium falciparum using channel color space segmentation method. 2017 th International Conference on Cyber and IT Service Management (CITSM); 2017 8-10 Aug. 2017. 36. Bibin D, Nair MS, Punitha P. Malaria Parasite Detection From Peripheral Blood Smear Images Using Deep Belief Networks. IEEE Access. 2017;5:9099-108.

37. Hung J, Goodman A, Lopes S, Rangel G, Ravel D, Costa F, et al. Applying Faster RCNN for Object Detection on Malaria Images. ArXiv. 2018;abs/1804.09548.

38. Bhuvan C, Bansal S, Gupta R, Bhan A, editors. Computer Based Diagnosis of Malaria in Thin Blood Smears Using Thresholding Based Approach. 2020 7th International Conference on Signal Processing and Integrated Networks (SPIN); 2020 27-28 Feb. 2020.

39. Kalkan SC, Sahingoz OK, editors. Deep Learning Based Classification of Malaria from Slide Images. 2019 Scientific Meeting on Electrical-Electronics \& Biomedical Engineering and Computer Science (EBBT); 2019 24-26 April 2019.

40. Shah D, Kawale K, Shah M, Randive S, Mapari R, editors. Malaria Parasite Detection Using Deep Learning : (Beneficial to humankind). 2020 4th International Conference on Intelligent Computing and Control Systems (ICICCS); 2020 13-15 May 2020.

41. Fuhad KMF, Tuba JF, Sarker MRA, Momen S, Mohammed N, Rahman T. Deep Learning Based Automatic Malaria Parasite Detection from Blood Smear and its Smartphone Based Application. Diagnostics (Basel). 2020;10(5):329.

42. Dong Y, Jiang Z, Shen H, Pan WD, Williams LA, Reddy VVB, et al., editors. Evaluations of deep convolutional neural networks for automatic identification of malaria 
infected cells. 2017 IEEE EMBS International Conference on Biomedical \& Health Informatics (BHI); 2017 16-19 Feb. 2017.

43. Vijayalakshmi A, Rajesh Kanna B. Deep learning approach to detect malaria from microscopic images. Multimedia Tools and Applications. 2020;79(21):15297-317.

44. Redmon J. Darknet: Open Source Neural Networks in C 2013--2016 [Available from: http://pjreddie.com/darknet/.

45. He K, Zhang X, Ren S, Sun J. Deep Residual Learning for Image Recognition. 2016 IEEE Conference on Computer Vision and Pattern Recognition (CVPR). 2016:770-8.

46. Huang G, Liu Z, Weinberger KQ. Densely Connected Convolutional Networks. 2017 IEEE Conference on Computer Vision and Pattern Recognition (CVPR). 2017:2261-9.

47. Elgendi M, Nasir MU, Tang Q, Fletcher RR, Howard N, Menon C, et al. The Performance of Deep Neural Networks in Differentiating Chest X-Rays of COVID-19 Patients From Other Bacterial and Viral Pneumonias. Frontiers in Medicine. 2020;7(550).

48. Jaiswal A, Gianchandani N, Singh D, Kumar V, Kaur M. Classification of the COVID-19 infected patients using DenseNet201 based deep transfer learning. J Biomol Struct Dyn. 2020:1 8.

49. Yurayart N, Kaewthamasorn M, Tiawsirisup S. Vector competence of Aedes albopictus (Skuse) and Aedes aegypti (Linnaeus) for Plasmodium gallinaceum infection and transmission. Vet Parasitol. 2017;241:20-5.

50. Veerayuth Kittichai, Theerakamol Pengsakul, Kemmapon Chumchuen, Yuttana Samang, Patchara Sriwichai, Natthaphop Phatthamolrat, Teerawat Tongloy, Komgrit Jaksukam, Santhad Chuwongin, Siridech Boonsang. Deep learning approaches for challenging species and gender identification of mosquito vectors. Scientific Reports. 2021;Accepted for publication. 51. Jiram AI, Ooi CH, Rubio JM, Hisam S, Karnan G, Sukor NM, et al. Evidence of asymptomatic submicroscopic malaria in low transmission areas in Belaga district, Kapit division, Sarawak, Malaysia. Malar J. 2019;18(1):156.

52. Raman J, Gast L, Balawanth R, Tessema S, Brooke B, Maharaj R, et al. High levels of imported asymptomatic malaria but limited local transmission in KwaZulu-Natal, a South African malaria-endemic province nearing malaria elimination. Malar J. 2020;19(1):152. 53. Torres K, Bachman CM, Delahunt CB, Alarcon Baldeon J, Alava F, Gamboa Vilela D, et al. Automated microscopy for routine malaria diagnosis: a field comparison on Giemsa-stained blood films in Peru. Malar J. 2018;17(1):339.

54. Rajaraman S, Jaeger S, Antani SK. Performance evaluation of deep neural ensembles toward malaria parasite detection in thin-blood smear images. PeerJ. 2019;7:e6977.

55. Sheeba F. TR, Mammen J.J., Nagar A.K., editor Detection of Plasmodium Falciparum in Peripheral Blood Smear Images. Proceedings of Seventh International Conference on BioInspired Computing: Theories and Applications (BIC-TA 2012); 2013; India: Springer. 56. Fuhad KMF, Tuba JF, Sarker MRA, Momen S, Mohammed N, Rahman T. Deep Learning Based Automatic Malaria Parasite Detection from Blood Smear and its Smartphone Based Application. Diagnostics (Basel). 2020;10(5). 

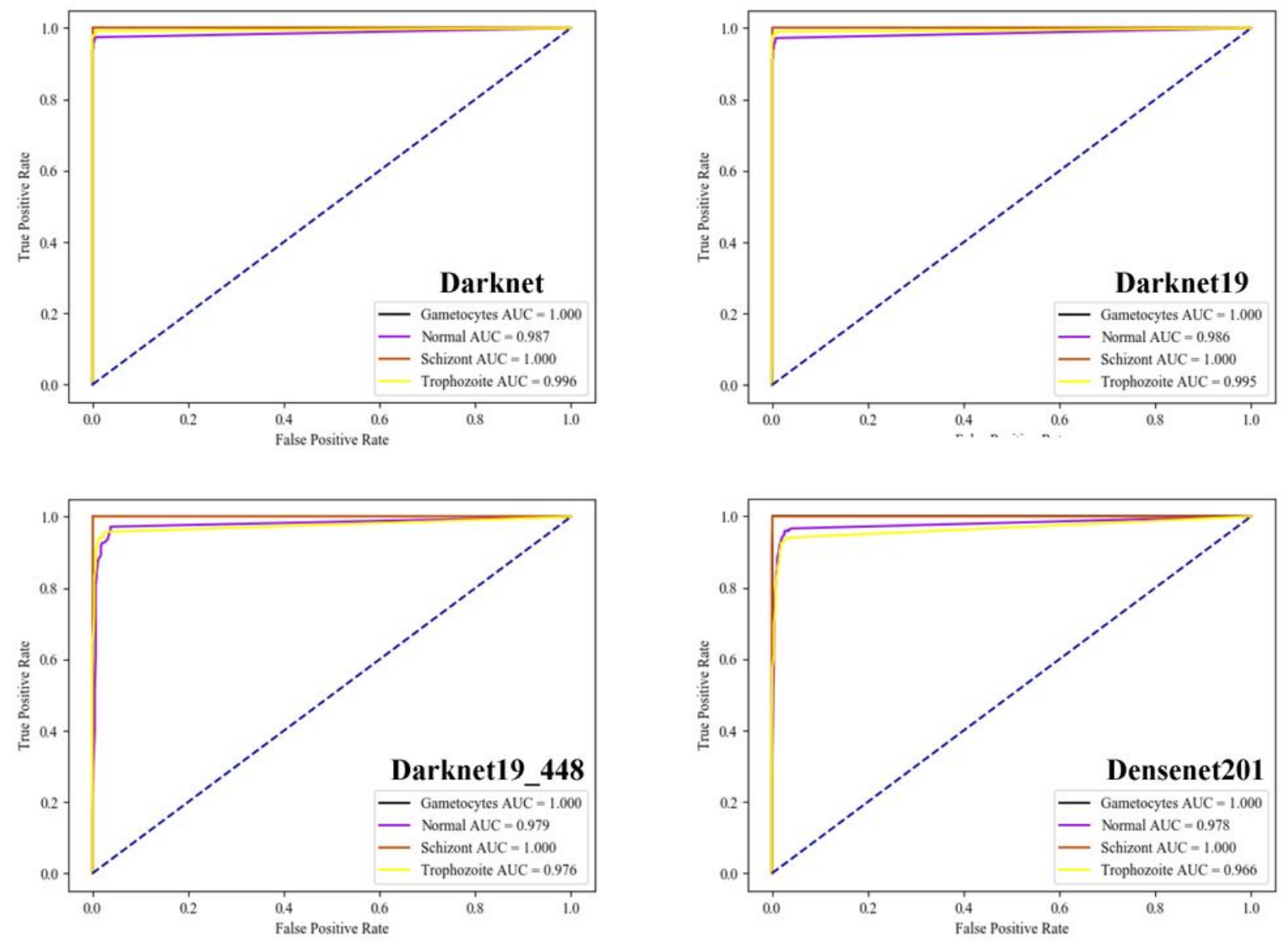

\section{Figure 1}

Prediction of the outcome of selectable models. The color of the guide map indicated the field of interest predicted by the model. The X-axis is the raw picture as input and models such as Darknet, Darknet19, Darknet19_448 and Densenet201. Y-axes are individual chicken-blood cells that are regular and those that are infected malaria, including trophozoites, schizonts and gametocytes. 


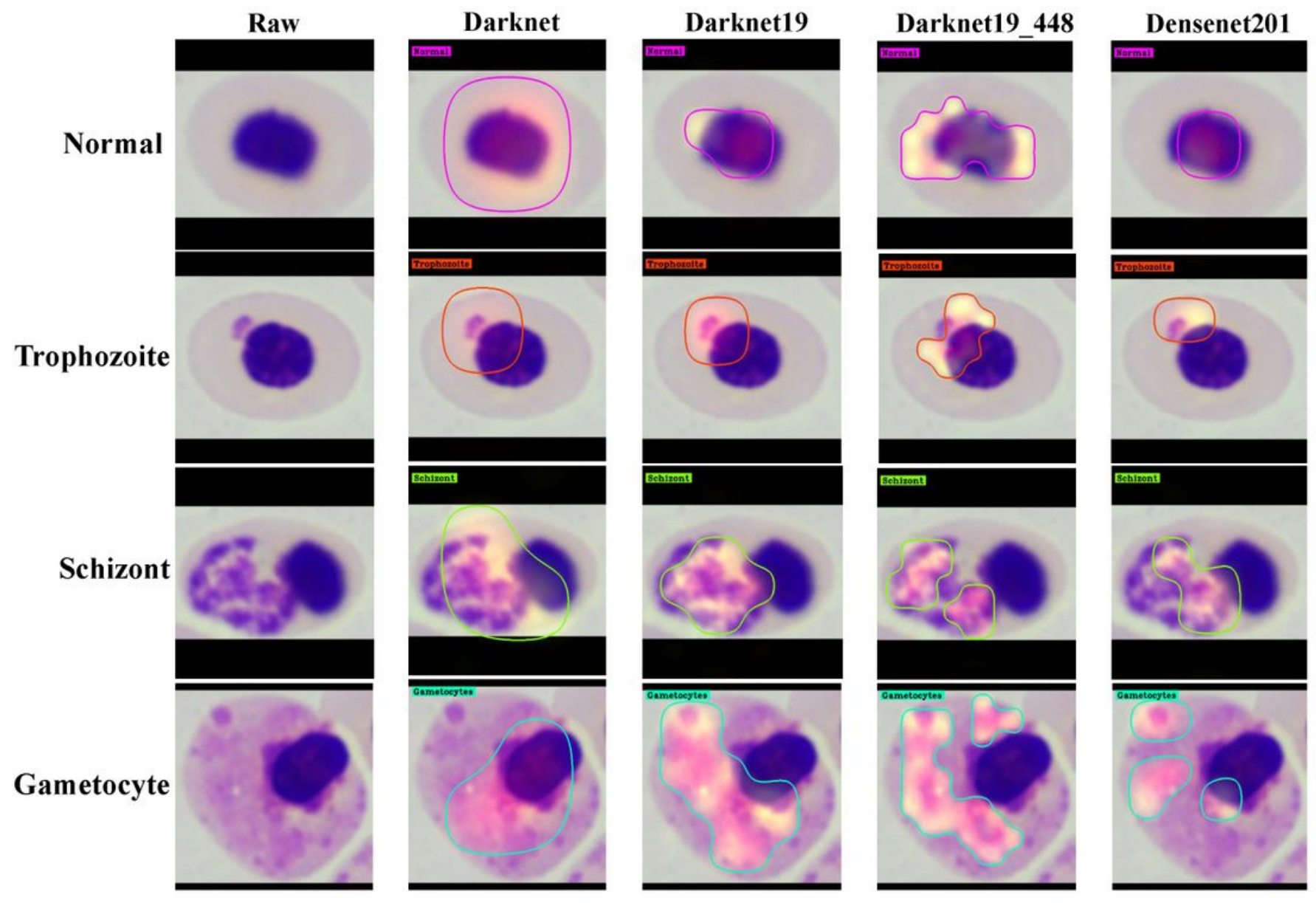

Figure 2

The schematic of the deep learning classification of the Darknet19 neural network model. Filters each convolution layer help generate feature map in the part of feature extraction. Guide map of the JET color gradient is used to visualize the input based on optimal weight. 


\section{$\underline{\text { Classification }}$}

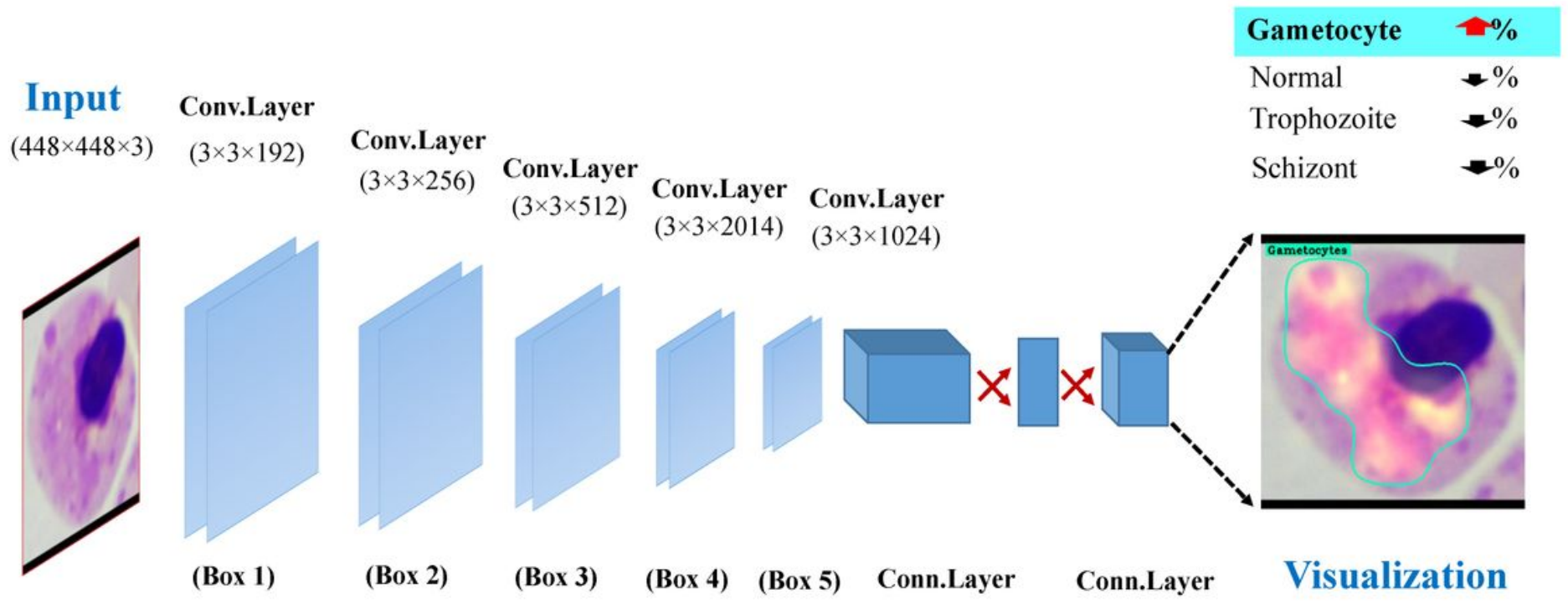

Figure 3

Comparison of the model- and class-wise performance based on AUC under the ROC curve 